\title{
PLANTAS MEDICINAIS: CURA SEGURA?
}

\author{
Valdir F. Veiga Junior* e Angelo C. Pinto
}

Instituto de Química, Universidade Federal do Rio de Janeiro, CT, Bloco A, Cidade Universitária, Ilha do Fundão, 21945-970 Rio de Janeiro - RJ

\section{Maria Aparecida M. Maciel}

Departamento de Química, Universidade Federal do Rio Grande do Norte, Campus Universitário, 59078-970 Natal - RN

Recebido em 7/6/04; aceito em 15/10/04; publicado na web em 28/2/05

\begin{abstract}
MEDICINAL PLANTS: SAFE CURE? This paper reviews the recent literature on synergism, adulteration and risks of using medicinal plants. The use of copaiba and sacaca plants as well as their adulteration and side effects, are also described. In addition, the new regulations on phytotherapeutic registration in Brazil and Europe are discussed.
\end{abstract}

Keywords: Copaifera sp; Croton cajucara; medicinal plants toxicity.

\section{INTRODUÇÃO}

A utilização de plantas com fins medicinais, para tratamento, cura e prevenção de doenças, é uma das mais antigas formas de prática medicinal da humanidade. No início da década de 1990, a Organização Mundial de Saúde (OMS) divulgou que 65-80\% da população dos países em desenvolvimento dependiam das plantas medicinais como única forma de acesso aos cuidados básicos de saúde ${ }^{1}$.

Ao longo do tempo têm sido registrados variados procedimentos clínicos tradicionais utilizando plantas medicinais. Apesar da grande evolução da medicina alopática a partir da segunda metade do século XX, existem obstáculos básicos na sua utilização pelas populações carentes, que vão desde o acesso aos centros de atendimento hospitalares à obtenção de exames e medicamentos. Estes motivos, associados com a fácil obtenção e a grande tradição do uso de plantas medicinais, contribuem para sua utilização pelas populações dos países em desenvolvimento.

Atualmente, grande parte da comercialização de plantas medicinais é feita em farmácias e lojas de produtos naturais, onde preparações vegetais são comercializadas com rotulação industrializada. Em geral, essas preparações não possuem certificado de qualidade e são produzidas a partir de plantas cultivadas, o que descaracteriza a medicina tradicional que utiliza, quase sempre, plantas da flora nativa.

É cada vez mais freqüente o uso de plantas medicinais das medicinas tradicionais indú e chinesa, completamente desconhecidas dos povos ocidentais. Estas plantas são comercializadas apoiadas em propagandas que prometem "benefícios seguros, já que se trata de fonte natural". Muitas vezes, entretanto, as supostas propriedades farmacológicas anunciadas não possuem validade científica, por não terem sido investigadas, ou por não terem tido suas ações farmacológicas comprovadas em testes científicos pré-clínicos ou clínicos.

Nos países em desenvolvimento, bem como nos mais desenvolvidos, os apelos da mídia para o consumo de produtos à base de fontes naturais aumentam a cada dia. Os ervanários prometem saúde e vida longa, com base no argumento de que plantas usadas há milênios são seguras para a população.

*e-mail: junior@iq.ufrj.br
Nos Estados Unidos e na Europa há mais controle no registro e na comercialização dos produtos obtidos de plantas. Nesses países, as normas para a certificação e o controle de qualidade de preparações vegetais são mais rígidos.

Pesquisa realizada nos EUA no ano de 1997 mostrou que 42\% da população haviam feito uso de plantas medicinais, pelo menos uma vez no ano de 1996, em tratamentos médicos alternativos. Esse percentual é cerca de 33,8\% maior em relação ao ano de 1990, quando a mesma pesquisa foi realizada ${ }^{2}$.

$\mathrm{Na}$ Alemanha, onde se consome metade dos extratos vegetais comercializados em toda a Europa (cerca de US\$ 3,5 bilhões do total de US\$ 7 bilhões, ou US\$ 42,90 per capita, em valores de $1997)^{3}$, plantas medicinais são utilizadas pela população para tratar resfriados $(66 \%)$, gripe (38\%), doenças do trato digestivo ou intestinal (25\%), dores de cabeça (25\%), insônia (25\%), úlcera estomacal $(36 \%)$, nervosismo $(21 \%)$, bronquite $(15 \%)$, doenças de pele $(15 \%)$, fadiga e exaustão $(12 \%)^{3}$. Nesse mesmo país, foi verificado que a auto-medicação com preparações à base de plantas medicinais é muito comum. Durante o ano de 1997, 1,5 milhão de pessoas utilizaram ervas medicinais durante o tratamento alopático. Mais da metade destes pacientes não comunicaram esse uso ao médico. Há entre 600 e 700 ervas utilizadas terapeuticamente, "sozinhas" ou em combinação com outras ervas, vendidas em farmácias, drogarias, mercados e lojas especializadas em produtos naturais na Alemanha. Além da auto-medicação, 70\% dos clínicos-gerais alemães prescrevem as centenas de ervas licenciadas nesse país. Estimativas conservadoras apontam que o seguro-saúde pago pelo governo alemão gasta 1,7 bilhões de dólares por ano com o pagamento de prescrições médicas que contém preparações vegetais. Em 1998, o fitoterápico Gingko biloba estava presente em 5,4 milhões de prescrições médicas ${ }^{4}$.

No Brasil, as plantas medicinais da flora nativa são consumidas com pouca ou nenhuma comprovação de suas propriedades farmacológicas, propagadas por usuários ou comerciantes. Muitas vezes essas plantas são, inclusive, empregadas para fins medicinais diferentes daqueles utilizados pelos silvícolas. Comparada com a dos medicamentos usados nos tratamentos convencionais, a toxicidade de plantas medicinais e fitoterápicos pode parecer trivial. Isto, entretanto, não é verdade. A toxicidade de plantas medicinais é um problema sério de saúde pública. Os efeitos adversos dos fitomedicamentos, possíveis adulterações e toxidez, bem como a 
ação sinérgica (interação com outras drogas) ocorrem comumente. As pesquisas realizadas para avaliação do uso seguro de plantas medicinais e fitoterápicos no Brasil ainda são incipientes, assim como o controle da comercialização pelos órgãos oficiais em feiras livres, mercados públicos ou lojas de produtos naturais.

Este artigo aborda, em aspectos gerais, alguns casos de toxicidade de plantas medicinais recentemente divulgados, que representam um risco em potencial para a população e alerta para o uso indiscriminado de duas plantas medicinais amplamente utilizadas na Região Amazônica: Croton cajucara e Copaifera sp.

\section{PLANTAS MEDICINAIS, FITOTERÁPICOS E FITOFÁRMACOS}

A OMS define planta medicinal como sendo "todo e qualquer vegetal que possui, em um ou mais órgãos, substâncias que podem ser utilizadas com fins terapêuticos ou que sejam precursores de fármacos semi-sintéticos" ". A diferença entre planta medicinal e fitoterápico reside na elaboração da planta para uma formulação específica, o que caracteriza um fitoterápico. Segundo a Secretaria de Vigilância Sanitária, em sua portaria $n^{\text {0. }} 6$ de 31 de janeiro de 1995 , fitoterápico é "todo medicamento tecnicamente obtido e elaborado, empregando-se exclusivamente matérias-primas vegetais com finalidade profilática, curativa ou para fins de diagnóstico, com benefício para o usuário. É caracterizado pelo conhecimento da eficácia e dos riscos do seu uso, assim como pela reprodutibilidade e constância de sua qualidade. É o produto final acabado, embalado e rotulado. Na sua preparação podem ser utilizados adjuvantes farmacêuticos permitidos na legislação vigente. Não podem estar incluídas substâncias ativas de outras origens, não sendo considerado produto fitoterápico quaisquer substâncias ativas, ainda que de origem vegetal, isoladas ou mesmo suas misturas". Neste último caso encontrase o fitofármaco, que por definição "é a substância ativa, isolada de matérias-primas vegetais ou mesmo, mistura de substâncias ativas de origem vegetal".

Com relação aos fitoterápicos, existem pontos que merecem atenção especial e serão abordados neste trabalho enfocando a presença de substâncias "não identificadas", adulterantes, diluentes, ou simplesmente misturas com outros extratos vegetais. Neste último caso, existe a possibilidade do comprometimento da qualidade do fitoterápico, um assunto que vem sendo abordado recentemente em publicações científicas ${ }^{6,7}$.

No caso da comercialização popular de plantas medicinais, muitos cuidados (válidos até mesmo para plantas de uso milenar) são relevantes, tais como identificação errônea da planta (pelo comerciante e pelo fornecedor), possibilidades de adulteração (em extratos, cápsulas com o pó da espécie vegetal, pó da planta comercializado em saquinhos e garrafadas), interações entre plantas medicinais e medicamentos alopáticos (que possam estar sendo ingeridos pelo usuário da planta), efeitos de superdosagens, reações alérgicas ou tóxicas.

\section{REAÇÕES ALÉRGICAS CAUSADAS PELO USO DE ÓLEOS ESSENCIAIS E OUTROS INGREDIENTES OBTIDOS DE PLANTAS MEDICINAIS}

A hipersensibilidade é um dos efeitos colaterais mais comuns causado pelo uso de plantas medicinais ${ }^{8}$. Ela pode variar de uma dermatite temporária (comum, por exemplo, entre os fitoquímicos) até um choque anafilático. São muito comuns dermatites provocadas pelo contato com planta (DCP), Tabela 1. Esse efeito tem sido provocado, em grande parte, por cosméticos que apresentam, na sua formulação, extratos de plantas ou substâncias isoladas de fonte ve- getal. Neste caso, não apenas os usuários como também os profissionais que manipulam os cosméticos, podem contrair dermatoses ${ }^{9}$. Muitas dermatites e reações alérgicas são causadas por tratamentos denominados de aromaterapia, uma prática que se popularizou ao longo da década de 1990, que envolve o uso de óleos essenciais concentrados. Como exemplo, podem-se citar os efeitos alérgicos da cânfora e de misturas contendo óleos de lavanda e jasmim ${ }^{10}$.

\section{REAÇÕES TÓXICAS E EFEITOS ADVERSOS PROVOCADOS POR PLANTAS MEDICINAIS}

O uso milenar de plantas medicinais mostrou, ao longo dos anos, que determinadas plantas apresentam substâncias potencialmente perigosas. Do ponto de vista científico, pesquisas mostraram que muitas delas possuem substâncias potencialmente agressivas e, por esta razão, devem ser utilizadas com cuidado, respeitando seus riscos toxicológicos.

Como exemplos de efeitos tóxicos de substâncias presentes em plantas podem ser citados os efeitos hepatotóxicos de apiol, safrol (Figura 1), lignanas e alcalóides pirrolizidínicos; a ação tóxica renal que pode ser causada por espécies vegetais que contém terpenos e saponinas e alguns tipos de dermatites, causadas por espécies ricas em lactonas sesquiterpênicas e produtos naturais do tipo furanocumarinas ${ }^{11}$. Componentes tóxicos ou antinutricionais, como o ácido oxálico, nitrato e ácido erúcico estão presentes em muitas plantas de consumo comercial ${ }^{12}$. Diversas substâncias isoladas de vegetais considerados medicinais possuem atividades citotóxica ou genotóxica e mostram relação com a incidência de tumores ${ }^{13}$.

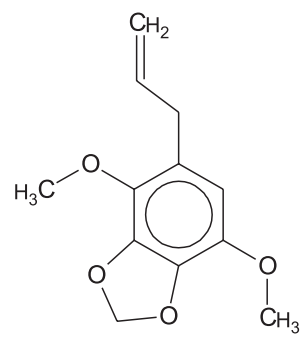

apiol

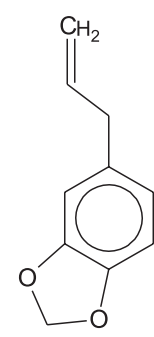

safrol
Figura 1. Estruturas do apiol e do safrol

O intenso apelo comercial advindo do forte movimento cultural dos naturalistas aqueceu, em todo o mundo, o consumo de plantas medicinais. Entretanto, não há respeito aos limites de uso dos fitoterápicos, não se fornecem informações sobre efeitos colaterais, e o consumo de plantas, do modo com vem sendo feito, representa cada vez mais um risco para a saúde humana. Estudos multidisciplinares, associando fitoquímicos e farmacólogos, tornam-se cada vez mais importantes para a definição dos potenciais terapêuticos e tóxicos de extratos vegetais ${ }^{14}$.

Um dos efeitos tóxicos relatados recentemente foi ocasionado pelo uso de cápsulas de têucrio (Teucrium chamaedrys L. - Labiateae), que causou uma epidemia de hepatite na França. A origem do efeito tóxico foi atribuída a diterpenos do tipo neo-clerodano, transformados pelo citocromo $\mathrm{P} 450$ em metabólitos hepatotóxicos, que apresentavam uma subunidade epóxido. Anteriormente, o uso do têucrio era tido como seguro até que a comercialização do vegetal em cápsulas associado à camomila, prescrito para dietas de emagrecimento, desencadeou os casos de hepatite tóxica ${ }^{15}$. Estudos farmacológicos mostraram que os diterpenóides furânicos (muitos estão presentes entre os clerodanos) causam apoptose dentro de $2 \mathrm{~h}$ em hepatócitos de ratos. Metabólitos eletrofílicos podem estimular a apoptose pela 
Tabela 1. Tipos de dermatites causadas por contato com plantas (DCP)*

\begin{tabular}{|c|c|c|c|}
\hline Tipos de DCP & Nome comum & Espécie & Família \\
\hline irritativas & $\begin{array}{l}\text { avelós esqueleto } \\
\text { cajueiro } \\
\text { comigo-ninguém-pode } \\
\text { juazeiro } \\
\text { melão-são-caetano }\end{array}$ & $\begin{array}{l}\text { Euphorbia tirucali } \\
\text { Anacardium occidentale } \\
\text { Dieffenbachia picta } \\
\text { Zizyphus joazeiro } \\
\text { Momordica charantia }\end{array}$ & $\begin{array}{l}\text { Euphorbiaceae } \\
\text { Anacardiaceae } \\
\text { Araceae } \\
\text { Rhamnaceae } \\
\text { Cucurbitaceae }\end{array}$ \\
\hline urticantes & cansanção & $\begin{array}{l}\text { Cnidosculus urens } \\
\text { Fleurya aestuans }\end{array}$ & $\begin{array}{l}\text { Euphorbiaceae } \\
\text { Urticaceae }\end{array}$ \\
\hline irritação mecânica & $\begin{array}{l}\text { buganvília } \\
\text { mandacaru } \\
\text { palma } \\
\text { xiquexique }\end{array}$ & $\begin{array}{l}\text { Bougainvillea spectabilis } \\
\text { Cereus jamacuru } \\
\text { Opuntia squamosus } \\
\text { Pilocereus gaunelei }\end{array}$ & $\begin{array}{l}\text { Nyctaginaceae } \\
\text { Cactaceae } \\
\text { Cactaceae } \\
\text { Cactaceae }\end{array}$ \\
\hline alérgicas & $\begin{array}{l}\text { alho } \\
\text { aroeira da praia } \\
\text { aroeira do sertão } \\
\text { caju } \\
\text { cebola } \\
\text { cebolinha verde } \\
\text { confrei } \\
\text { eucalipto } \\
\text { hortelã } \\
\text { manga }\end{array}$ & $\begin{array}{l}\text { Allium sativum } \\
\text { Schinus terebinthifolius } \\
\text { Astronium orindeuva } \\
\text { Anacardium occidentale } \\
\text { Allium cepa } \\
\text { Allium schoenoprasum } \\
\text { Symphytum officinale } \\
\text { Eucalyptus globulus } \\
\text { Menta villosa } \\
\text { Mangifera indica }\end{array}$ & $\begin{array}{l}\text { Liliaceae } \\
\text { Anacardiaceae } \\
\text { Anacardiaceae } \\
\text { Anacardiaceae } \\
\text { Liliaceae } \\
\text { Liliaceae } \\
\text { Boraginaceae } \\
\text { Myrtaceae } \\
\text { Labiatae } \\
\text { Anacardiaceae }\end{array}$ \\
\hline fitofotodermatites & $\begin{array}{l}\text { aipo } \\
\text { angélica } \\
\text { arruda } \\
\text { bergamota } \\
\text { canela } \\
\text { cenoura } \\
\text { figo } \\
\text { limão } \\
\text { mama cadela } \\
\text { salsa }\end{array}$ & $\begin{array}{l}\text { Anethum graveolens } \\
\text { Angelica archangelica } \\
\text { Ruta graveolens } \\
\text { Citrus bergamia } \\
\text { Cinnamomum zeylanicum } \\
\text { Daucus carota } \\
\text { Ficus carica } \\
\text { Citrus limonum } \\
\text { Brosimum gaudichaudii } \\
\text { Petroselinum sativum }\end{array}$ & $\begin{array}{l}\text { Umbelliferae } \\
\text { Umbelliferae } \\
\text { Rutaceae } \\
\text { Rutaceae } \\
\text { Lauraceae } \\
\text { Umbelliferae } \\
\text { Moraceae } \\
\text { Rutaceae } \\
\text { Moraceae } \\
\text { Umbelliferae }\end{array}$ \\
\hline
\end{tabular}

* Nem todas as plantas citadas nesta tabela são medicinais

captura de tióis, aumento da concentração de cálcio e ativação das enzimas transglutaminase e endonuclease dependentes de cálcio ${ }^{16}$. Em países como a Inglaterra, o têucrio era constantemente utilizado, sem aviso aos consumidores, para substituir extratos de escutelária (Scutellaria lateriflora) em associações com valeriana. A mistura tóxica levou erroneamente à crença de que tanto a valeriana quanto a escutelária poderiam ser tóxicas quando em misturas, por efeito sinérgico ${ }^{17}$.

Outro caso importante é o do confrei (Symphytum officinale L.). Esta planta é utilizada na medicina tradicional como cicatrizante devido à presença da alantoína, mas também possui alcalóides pirrolizidínicos (Figura 2), os quais são comprovadamente hepatotóxicos e carcinogênicos ${ }^{18}$. Após diversos casos de morte ocasionados por cirrose resultante de doença hepática veno-oclusiva, desencadeadas por estes alcalóides, o uso do confrei foi condenado pela OMS

Outras plantas medicinais são potencialmente perigosas, podendo-se citar as espécies do gênero Senecio ${ }^{18}$, a jurubeba (Solanum paniculatum L.), ipeca (Cephaelis ipecacuanha (Brot.) A. Rich.) e arnica (Arnica montana L.), que podem causar irritação gastrointestinal; o mastruço (Chenopodium ambrosioides L.) e a trombeteira (Datura suaveolens Humb. \& Bopl ex Willd.), que podem lesionar o sistema nervoso central; o cambará (Lantana camara L.), conhecido por sua hepatotoxicidade; a cáscara-sagrada<smiles>[R]OCC1=CCN2CCC(O[R])C12</smiles><smiles>NC(=O)NC1NC(=O)NC1=O</smiles>

estrutura básica de alcalóides pirrolizidínicos

alantoína

Figura 2. Alantoína e estrutura básica dos alcalóides pirrolizidínicos do confrei

(Rhamnus purshiana DC), que causa distúrbios gastro-intestinais (como diarréia grave) ${ }^{19}$ e a arruda (Ruta graveolens), que pode provocar aborto, fortes hemorragias, irritação da mucosa bucal e inflamações epidérmicas ${ }^{20}$. Em doses elevadas, até mesmo o jatobá (Hymenaea courbail L.), conhecido como expectorante e fortificante, pode desencadear reações alérgicas, e a sucuúba (Himathantus sucuuba (Spruve) Woodson), usada no combate à amebíase, úlcera e gastrite, pode ser abortiva ${ }^{21}$. No caso de gestantes, o uso de espécies vegetais deve seguir rigorosamente os mesmos cuidados dos medicamentos alopáticos. Entre as plantas medicinais que podem causar riscos para mulheres grávidas, por estimular a motilidade uterina e provocar aborto, encontram-se alho (Allium sativum), aloe (Aloe ferox), angélica (Angelica 
Tabela 2. Efeitos adversos que podem ocorrer pelo uso de plantas medicinais

\begin{tabular}{llll}
\hline Nome popular & Espécie & Efeitos adversos(toxicidade) & Constituintes responsáveis \\
\hline Alho & Allium sativum(Liliaceae) & Nauseas, vômitos, dermatite & Compostos à base de enxofre \\
& por contato & Antraquinonas \\
Aloe & Aloe ferox(Liliaceae) & Desconforto abdominal & Furanocumarinas \\
Angélica & Angelica archangelica(Umbelliferae) & Fotodermatite & Anetol \\
Anis & Pimpinella anisum(Umbelliferae) & Dermatite por contato & Óleo volátil (ascaridol) \\
Boldo & Peumus boldo(Monimiaceae) & Irritação renal & Capsaicinóides \\
Capsicum & Capsicum annum(Solanaceae) & Alveolite alérgica & Cinamaldeído \\
Cássia & Cinnamomum cassia $($ Lauraceae) & Reações alérgicas & Alcalóides pirrolizidínicos \\
Confrei & Symphytum officinale(Boraginaceae) & Hepatotoxicidade & Lactonas sesquiterpênicas \\
Dente-de-leão & Taraxacum officinale(Compositae) & Reações alérgicas por contato & Hipericina \\
Erva-de-São-João & Hypericum perforatum(Guttiferae) & Fotodermatite & Resina \\
Guaiacum & Guaiacum officinale(Zygophyllaceae) & Dermatite por contato & Xantinas \\
Mate & Ilex paraguaiensis(Aquifoliaceae) & Distúrbios hepáticos & Antraquinonas \\
Sene & Cassia angustifolia(Leguminoseae) & Desconforto abdominal, perda & \\
& & de eletrólitos e água & \\
\hline
\end{tabular}

archangelica), arnica (Arnica montana), cânfora (Cinnamomum canphora), confrei (Symphitum officinalis), eucalipto (Eucaliptus globulus), alecrim (Rosmarinus officinalis), gengibre (Zengiber officinalis) e sene (Cassia angustifolia e Cassia acutifolia).

Na Tabela 2 encontram-se outros exemplos de substâncias tóxicas presentes em plantas medicinais ${ }^{22}$.

Alguns óleos essenciais também devem ser evitados, como exemplo, os provenientes de bétula (Betula alba), cedro (Cedrela brasiliensis), erva-doce (Pimpinella anisum), jasmim (Jasminum officinalis), manjericão (Origanum basilicum), manjerona (Majorana hortensis), tomilho (Thymus vulgaris), rosa (Rosa sp.) e lavanda (Lavanda angustifolia). Neste último caso, deve-se evitar o consumo, especialmente nos primeiros meses de gravidez ${ }^{22}$.

Estudo recentes, realizados com ratas grávidas, apontaram o efeito colateral abortivo da espinheira-santa (Maytenus ilicifolia), planta medicinal de comprovada baixa toxicidade ${ }^{23}$ e ação antiulcerogênica ${ }^{24,25}$, anti-inflamatória e antinoniceptiva ${ }^{25}$. Extratos hidroalcoólicos dessa planta mostraram-se abortivos por atuarem no período de pré-implantação dos embriões no útero ${ }^{26}$.

Também há riscos para os lactentes (exemplificadas na Tabela 3), associados ao consumo de plantas medicinais pela mãe durante o aleitamento $^{22}$.

\section{PRINCIPAIS CONTAMINANTES DE PLANTAS MEDICINAIS}

\section{Metais pesados}

Metais pesados fazem parte das preparações farmacêuticas de várias escolas da medicina oriental, utilizados geralmente em conjunto com extratos de plantas medicinais. Na medicina tradicional indiana a importância de metais, como ouro, cobre, estanho, chumbo, mercúrio, ferro, prata e zinco, é enfatizada para a bioquímica do corpo humano. Na medicina tradicional chinesa, mercúrio faz parte de algumas preparações sob a terminologia 'cinnabaris' (sulfeto de mercúrio), 'calomel' (cloreto de mercúrio) ou 'hydrargyri oxydum rubrum' (óxido de mercúrio). Estes produtos são utilizados para indicações variadas, como tranqüilizantes, anti-epiléticos, no tratamento de úlceras e insônia. O chumbo é utilizado como 'Mi Tuo Seng' (Lithargyrum) e o arsênio como 'Xiong Huang' (Realgar). No entanto, alguns livros-texto como os Aiurvédicos, alertam para a toxicidade desses metais e apresentam alguns métodos para a sua detoxificação ${ }^{27}$.
Tabela 3. Riscos para o lactente pela contaminação da mãe, proveniente de fármacos presentes em plantas medicinais

\begin{tabular}{ll}
\hline Composição do fármaco & Efeito Biológico \\
\hline Salicilatos & Tendência à hemorragia \\
Bases purínicas & Taquicardia \\
Antraquinonas & Diarréia \\
Lactonas sesquiterpênicas & Desnutrição (por rejeição ao leite \\
& devido ao gosto desagradável) \\
Alcalóides pirrolizidínicos & Hepatotoxicidade \\
Alcalóides indólicos & Vômito, diarréia, câimbras, obstrução \\
& nasal \\
\hline
\end{tabular}

A contaminação de vegetais com metais pode ter diversas origens, tais como acidental, proposital, contaminação do solo, de materiais de origem natural ou mineral e durante a manufatura ${ }^{27}$.

Um grande risco para a população, em geral, são os fitoterápicos importados de países asiáticos, uma vez que as formulações são bastante diferentes das preparadas pelos ocidentais, contendo metais pesados em concentrações que, muitas vezes, ultrapassam os valores seguros para consumo. Um estudo realizado em Londres no período entre 1991 e 1995 mostrou uma série de efeitos adversos associados à medicina tradicional oriental. De 12 casos de contaminação por mercúrio, chumbo e arsênio, 9 foram associados a ervas medicinais e cosméticos indús ${ }^{28}$.

Pesquisadores indianos avaliaram recentemente a concentração de metais pesados em 31 formulações Aiurvédicas. Com exceção de uma dessas preparações, todas excederam os limites legais de metais pesados, sendo que 16 delas ultrapassaram por mais de duas ordens de grandeza os valores máximos permitidos (no caso do mercúrio, $1 \mathrm{ppm}$ ). Várias formas diferentes de mercúrio foram encontradas nessas preparações ${ }^{29}$. Numerosos casos de envenenamento por metais pesados associados ao uso da Medicina Tradicional Chinesa (MTC) têm sido publicados nos últimos anos. O chumbo é um dos principais responsáveis por estes envenenamentos. Outros metais pesados, como mercúrio, arsênio, cádmio, cobre e tálio também têm sido encontrados em fitoterápicos da $\mathrm{MCT}^{30}$.

Estudos realizados em Singapura com 2080 amostras de medicamentos chineses revelaram que 42 amostras excediam os limites permitidos para metais pesados. Destas, 28 continham mercúrio, 8 chumbo, 6 arsênio e 1 cobre $^{31}$.

Estudos realizados nos Estados Unidos mostraram que, em 251 
produtos da MTC, $24 \%$ continham pelo menos $10 \mathrm{ppm}$ de chumbo, $36 \%$, uma média de 14,6 ppm de arsênio; $35 \%$, uma média de 1046 ppm de mercúrio e $23 \%$, mais de um elemento contaminante e/ou adulterante ${ }^{32}$.

Estudos recentes, realizados no Brasil com plantas de origem nacional e outras de diversas origens, mostraram a presença de metais em altas concentrações. Em extratos de Aesculus hippocastanum, obtidos na França e Alemanha, chumbo foi detectado na concentração de $1.480 \mathrm{mg} / \mathrm{g}$ de extrato, $440 \%$ acima da dose máxima recomendada ${ }^{33}$.

Os riscos de contaminação com metais pesados em formulações contendo extratos de plantas medicinais orientais têm aumentado significativamente com a possibilidade de aquisição de medicamentos via Internet.

\section{Contaminações com fármacos ou outras plantas medicinais}

Além dos metais pesados, têm sido encontrados fármacos, adicionados com o objetivo de aumentar os efeitos propagados, em fitoterápicos da chamada Medicina Tradicional Chinesa ${ }^{32}$. De 251 amostras da MTC analisadas nos EUA, em 7\% foram encontradoas produtos contendo fármacos não declarados, como efedrina, clorfeniramina, metiltestosterona e fenacetina ${ }^{32}$. Além destes, aminopirina, aspirina, betametasona, clordiazepóxido, clorfeniramina, corticosteróis, diclofenaco, indometacina, paracetamol, fenacetina, fenilbutasona, teofilina, ácido mefenâmico e diazepam também já foram encontrados em produtos da MTC associados a distúrbios renais agudos e nefrite intersticial ${ }^{34}$.

Várias mortes foram associadas, nos EUA, ao uso do fitoterápico Ma Huang (Ephedra sinica) originário da MTC. Esse fitoterápico contém efedrina $^{35}$, efedradinas e outros constituintes não alcaloidais, como leucoantocianidinas e também flavonóides ${ }^{10}$. Na Inglaterra ${ }^{36}$ foram registrados casos de psicose em pacientes com paranóia e alucinações visuais naqueles que tomaram Ma Huang durante dez dias consecutivos. Apesar disso, revistas britânicas continuam anunciando o Ma Huang como sendo um tônico natural que acalma a mente, estimula o corpo e, ainda, induz a perda de peso ${ }^{36}$. Nos Estados Unidos, 15 mortes de adolescentes foram atribuídas ao consumo de Ma Huang, comercializado com nomes de "Herbal Ecstasy" e "Ultimate Xphoira". $\mathrm{O}$ produto, além de ter boa vendagem via Internet, é vendido como afrodisíaco em eventos de rock. No entanto, trata-se de um coquetel de fármacos e ervas com efeitos adversos desconhecidos. Geralmente, esses produtos contêm outros fármacos estimulantes, como a cafeína. Efedrina, um dos produtos ativos do Ma Huang, era vendido como descongestionante nasal para pacientes com rinite, como broncodilatador e também em dietas de emagrecimento. Suas propriedades biológicas são semelhantes às da anfetamina, a qual causa nervosismo, taquicardia e psicose ${ }^{35}$. Espécies de Ephedra, devido à presença de efedrinas, podem provocar perda de memória, miopatia, neuropatia, palpitação, hipertensão e psicose ${ }^{35}$.

A presença de esteróis como a fluocortolona e a prednisolona foi detectada em cosméticos da MTC, em cremes para o tratamento de eczema, contudo, somente os cremes contendo até $1 \%$ de hidrocortisona são permitidos pelas leis britânicas, constituindo não somente uma adulteração, mas também venda de produto não autorizado pelas leis locais, camuflado em planta medicinal ${ }^{37}$.

A contaminação de fármacos da MTC com extratos de plantas tóxicas é outro problema na comercialização segura de fitoterápicos. Na Bélgica, o uso de fitoterápicos da MTC contaminados com Aristolochia fangchi causou uma epidemia de nefropatia intestinal subaguda. A biópsia de rins e uretras retirados de 19 pacientes mostrou, em $40 \%$ deles, sinais conclusivos de neoplasmas ${ }^{38}$. Um levantamento posterior mostrou que metade dos 80 pacientes precisou de transplantes renais ${ }^{39}$. Plantas da família Aristolochiaceae podem conter ácidos aristolóquicos e, por isso, devem ser evitadas na fitoterapia. Existem pelo menos 14 diferentes ácidos aristolóquicos, que são potentes agentes nefrotóxicos e carcinogênicos (câncer na uretra) ${ }^{40}$.

Estudos recentes demonstraram que outros fitoterápicos da MTC, como o popular Bajiaolian, têm apresentado efeitos tóxicos. O Bajiaolian causou diversos casos de danos ao fígado e trombocitopenia devido à presença da substância tóxica podofilotoxina ${ }^{41}$. A erva conhecida como Gwai-Kou (Podophylum hexandrum) tem aparecido como adulterante de outras ervas, como o Lung-Dam-Cho (Gentiana $s p$.) e o Wai-Ling-Sin, que é a raiz de diversas espécies de Clematis $s p$., levando a casos de neuropatia, encefalopatia e nefropatia. Autoridades de Hong Kong avaliaram 234 amostras de fitoterápicos e encontraram Podophylum em 22 delas $^{42}$. Podofilotoxinas e lignanas relacionadas, encontradas em espécies de Podophylum, causam danos às fibras nervosas dos sistemas nervoso central e periférico, bem como aos neurônios dos gânglios dorsais ${ }^{43,}{ }^{44}$. Existem outros fitoterápicos da MTC que provocam hepatoxicidade. Sete casos de hepatite aguda foram relatados após o uso de um sedativo e analgésico conhecido como Jin Bu Huan, utilizado há mais de 1000 anos na $\mathrm{MTC}^{39}$. Apesar do produto relacionado ao Jin Bu Huan ser Poligala chinensis, a análise de cápsulas contendo esse produto apresentou altas concentrações de tetraidropalmatina, uma substância encontrada em altas concentrações em espécies do gênero Stephania e ausente em Poligala chinenses.

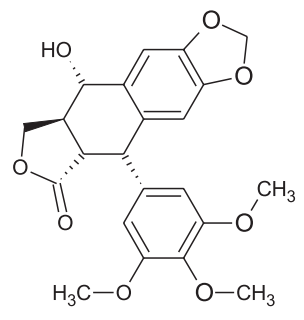

podofilotoxina

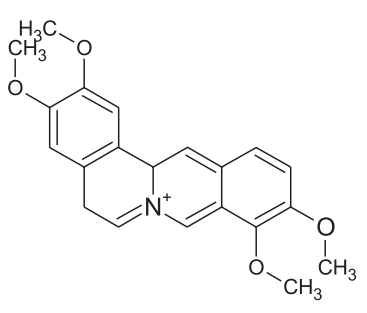

palmatina
Figura 3. Estruturas da podofilotoxina e da palmatina

\section{Outras contaminações}

Níveis altos de organofosforados foram recentemente encontrados em amostras de cumina (Cuminum cyminum), uma planta amplamente consumida por crianças e recém-nascidos para acalmar tosses e aliviar dores de garganta ${ }^{45}$. Relatos científicos mostraram que compostos organofosforados do tipo profenofos causam mal estar, dores de estômago e diarréia ${ }^{45}$. No caso do uso da cumina, que não é classificada nem como alimento nem como droga, não existe respaldo para sua utilização pela legislação atual ${ }^{46}$.

A possibilidade de contaminações microbiológicas também deve ser atentamente observada, em especial em plantas medicinais vendidas em feiras e mercados populares, como ocorre comumente no Brasil. Na Europa, verificou-se que, de um total de 138 amostras provenientes de 31 tipos de fitoterápicos obtidos de 9 diferentes fornecedores da Áustria e da Alemanha, 4 estavam contaminadas com Escherichia coli, 2 com Campylobacter jejuni e 9 possuíam potenciais produtores de aflatoxinas, os quais foram identificados como sendo fungos aflatoxigênicos ${ }^{47}$.

\section{INTERAÇÕES COM FÁRMACOS SINTÉTICOS E EFEITOS SINÉRGICOS}

Um exemplo clássico de efeito sinérgico encontra-se nos cons- 
tituintes da papoula (Papaver somniferum). Entre os mais de 30 alcalóides dessa espécie, a morfina apresenta ação analgésica e a papaverina, efeito vasodilatador (Figura 4$)^{48}$.

A warfarina é um dos fármacos mais empregados como anticoagulante. Sua ação pode ser antagonizada ou potencializada pelo emprego de um grande número de ervas ${ }^{49}$, dentre as quais a angélica (Angelica archangelica), cujas cumarinas apresentam propriedade anti-coagulante, e a agrimonia (Agrimonia eupatoria), com propriedades coagulantes.

Deve-se levar em conta, também, que muitos medicamentos sintéticos ou semi-sintéticos têm origem em plantas medicinais e são metabolizados nas mesmas substâncias no organismo, como é o caso do ácido acetilsalicílico (aspirina) e a salicilina, extraída de Salix alba. Ambos são transformados no fígado em ácido salicílico. Logo, extratos dessa planta podem apresentar efeitos semelhantes aos da aspirina, aumentando o risco de hemorragia nos tratamentos utilizando warfarina ${ }^{50}$.

Fitoterápicos à base de Salix são comercializados como analgésicos "sem-aspirina", em especial para crianças sensíveis ao ácido acetilsalicílico. Salicilatos não são recomendados para pessoas com menos de 19 anos, em especial para crianças entre 4 e 12 anos, que podem, assim, desenvolver a síndrome de Reye, doença muitas vezes fatal, que causa danos irreversíveis ao cérebro e ao fígado ${ }^{50}$.
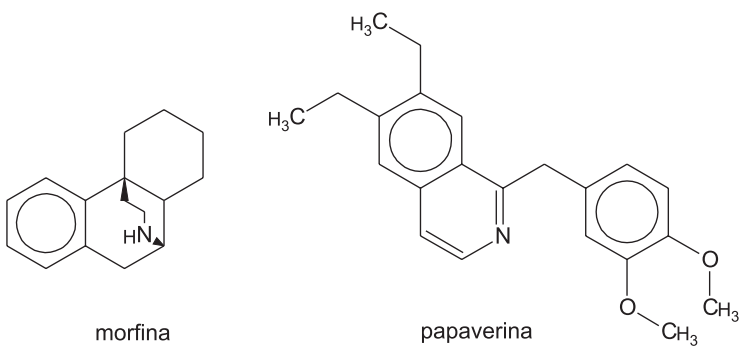

Figura 4. Estruturas de alcalóides de Papaver somniferum

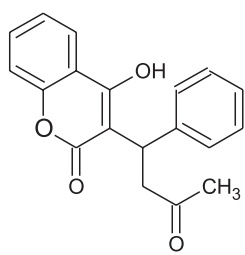

warfarina

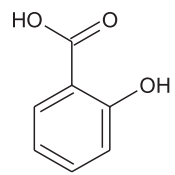

ácido salicílico
Figura 5. Estruturas da warfarina e do ácido salicílico

A administração de dente-de-leão (Taraxacum officinales) pode potencializar a atividade de diuréticos sintéticos, em especial em pacientes idosos hipertensos. $\mathrm{O}$ ginseng (Panax ginseng) pode alterar a pressão arterial e a raiz-doce (alcaçuz-da-europa, Glycirrhiza glabra) pode gerar uma atividade mineralocorticóide nesses pacientes ${ }^{50}$.

Qualquer erva com propriedades cardiotônicas ou hipertensivas pode agir sinergicamente com fármacos vasodilatadores das coronárias à base de nitratos (como o dinitrato de isosorbida) e com bloqueadores dos canais de cálcio (como a nifedipina) ${ }^{50}$.

Ervas sedativas que atuam no sistema nervoso central, como o maracujá (Passiflora officinalis) e a valeriana (Valeriana officinalis), podem interagir com hipnóticos e ansiolíticos ${ }^{50}$.

Os extratos de alho, já há muito conhecidos da medicina popular, têm sido bastante utilizados nos últimos anos para redução de níveis elevados de colesterol, um dos efeitos colaterais provocados por alimentos gordurosos, estresse e também, em casos mais drásticos, por medicamentos usados por portadores do vírus HIV ${ }^{51}$. A associação entre extratos à base de alho e medicamentos como o saquinavir (Fortovase ${ }^{\circledR}$ ) pode levar a interações perigosas uma vez que, tanto o alho quanto os inibidores de proteases, utilizados no coquetel de medicamentos anti-HIV, atuam na mesma via metabólica, conhecida como sistema enzimático do citocromo P450. Estudos científicos comprovaram que, na presença de suplementos à base de alho, os níveis plasmáticos do saquinavir diminuíram cerca de $50 \%$

Pacientes epilépticos tratados com fenotiazínicos devem evitar o consumo de óleo de prímula (Oenothera biennis), que pode provocar um quadro de epilepsia do lóbulo temporal ${ }^{51}$.

Ervas antidepressivas, como a erva-de-São-João (Hypericum perforatum), podem interferir na atividade antidepressiva de fármacos sintéticos. Essa erva representa um exemplo de planta medicinal largamente utilizada no mundo inteiro e, por esta razão, é alvo de variados estudos ${ }^{52}$. Alguns destes estudos estão relacionados a propriedades semelhantes às observadas para extratos à base de alho, que levam à diminuição dos níveis plasmáticos de inibidores de proteases, como o indinavir, bem como o aprenavir, nelfinavir, ritonavir e saquinavir. A erva-de-São-João pode apresentar efeito similar em inibidores de transcripase reversa nãonucleosídicos, como o delavirdina, efavirenze e nevirapine, que são metabolizados pela mesma via metabólica. Conseqüentemente, o uso desta erva é desaconselhado para a utilização em conjunto com inibidores de protease e de transcripase reversa não nucleosídicos, podendo resultar em perda da resposta virológica, desenvolvimento de resistência ou resistência-cruzada ${ }^{53}$. Muitos outros fármacos como, por exemplo, aqueles utilizados para doenças do coração (como digoxina, nifedipina, digitoxina diltiazem e betabloqueadores), depressão (imipramina, amoxapina e amitriptilina), certos tipos de câncer (ciclofosfamida, tamoxifen, taxol e etoposídeo), prevenção de rejeição de transplantes (ciclosporina, rapamicina e tacrolimus) e gravidez (etil estradiol) têm mecanismo semelhante. Alguns dos vários tipos de interação com drogas sintéticas são apresentados na Tabela $4^{52}$.

Algumas ervas medicinais, entretanto, podem interagir de forma positiva, aumentando os efeitos metabólicos. Alguns desses efeitos foram divulgados em publicações recentes (Tabela 5) ${ }^{54,55}$.

\section{FITOFARMACOLOGIA E AÇÃO TOXICOLÓGICA DE DUAS PLANTAS AMPLAMENTE COMERCIALIZADAS NA REGIÃO AMAZÔNICA DO BRASIL}

\section{Óleo de copaíba}

As copaibeiras são árvores comuns na América Latina, em especial no sudeste brasileiro e na região Amazônica. Pertencentes ao gênero Copaifera, contam com mais de 60 espécies catalogadas. Dessas árvores da família das Leguminosas-Caesalpiniaceas, é exudado, através de furo realizado no tronco, um óleo-resina chamado óleo de copaíba. Esse óleo é utilizado na medicina popular brasileira como anti-inflamatório das vias superiores e urinárias, tendo aplicação mais ampla como anti-séptico. No entanto, muitas outras aplicações farmacológicas são citadas para esse óleo. No Brasil seiscentista, o Padre José de Anchieta citava o óleo de copaíba como um potente cicatrizante. Atualmente, esse óleo é comercializado em farmácias e lojas de produtos naturais de todo o país, com indicações diversificadas ${ }^{56}$.

Estudos realizados com óleos de copaíba obtidos de todo o Brasil mostraram que esses óleos são misturas de sesquiterpenos e diterpenos. As concentrações e a natureza dos sesquiterpenos e 
Tabela 4. Estudos clínicos das interações entre a erva-de-São-João e outros fármacos

\begin{tabular}{|c|c|c|c|}
\hline Co-medicação & Resultados da interação & Mecanismo provável & Ocorrências \\
\hline Ciclosporina & $\begin{array}{l}\text { Diminuição dos níveis de ciclosporina/ } \\
\text { episódios de rejeição }\end{array}$ & Indução de enzimas hepáticas & 50 \\
\hline $\begin{array}{l}\text { Contraceptivos orais } \\
\text { (etilestradiol/desogestrel) }\end{array}$ & Interrupção do sangramento menstrual & Indução de enzimas hepáticas & 12 \\
\hline Teofilina & Diminuição dos níveis plasmáticos de teofilina & Indução de enzimas hepáticas & 1 \\
\hline Warfarina & $\begin{array}{l}\text { Diminuição dos níveis plasmáticos da } \\
\text { warfarina e do efeito anti-coagulante }\end{array}$ & Indução de enzimas hepáticas & 7 \\
\hline Amitriptilina & Diminuição dos níveis plasmáticos da amitriptilina & Indução de enzimas hepáticas & * \\
\hline Indinavir & Diminuição do nível plasmático do indinavir & $\begin{array}{l}\text { Indução de enzimas hepáticas; } \\
\text { Indução da P-Glicoproteína intestinal }\end{array}$ & $*$ \\
\hline Digoxina & Diminuição dos níveis plasmáticos da digoxina & Indução da P-Glicoproteína intestinal & * \\
\hline
\end{tabular}

* Interações demonstradas em estudos clínicos

Tabela 5. Interações entre preparações à base de ervas e fármacos da medicina convencional

\begin{tabular}{|c|c|c|}
\hline Erva & Fármaco convencional & Problema potencial \\
\hline $\begin{array}{l}\text { alcaçuz-da-Europa(Glycyrrhiza glabra }) \\
\text { Leguminoseae }\end{array}$ & Espironolactonas & Antagonismo do efeito diurético \\
\hline artemísia(Tanacetum parthenium)Asteraceae & $\begin{array}{l}\text { Anti-inflamatórios não-esteroidais } \\
\text { digoxina }\end{array}$ & $\begin{array}{l}\text { Inibição do efeito da erva } \\
\text { Interferência na farmacodinâmica e no } \\
\text { monitoramento do nível da droga no } \\
\text { organismo }\end{array}$ \\
\hline $\begin{array}{l}\text { erva-de-São-João(Hypericum perforatum) } \\
\text { Guttiferae }\end{array}$ & Inbidores de $\mathrm{MAO}$ & $\begin{array}{l}\text { Ausência de evidências de segurança de uso } \\
\text { concomitante }\end{array}$ \\
\hline $\begin{array}{l}\text { espinheira-santa(Maytenus ilicifolia) } \\
\text { Celastraceae }\end{array}$ & $\begin{array}{l}\text { Esteróides anabólicos, metotrexato, } \\
\text { amiodarona, cetoconazol } \\
\text { Imunosupressores }\end{array}$ & $\begin{array}{l}\text { Hepatotoxicidade } \\
\text { Efeitos antagonistas }\end{array}$ \\
\hline ginseng(Panax ginseng)Araliaceae & $\begin{array}{l}\text { Sulfato de fenelzina } \\
\text { Estrogênio e corticosteróides } \\
\text { Insulina, sulfaniluréias } \\
\text { digoxina }\end{array}$ & $\begin{array}{l}\text { Cefaléia, tremores } \\
\text { "Efeitos aditivos" } \\
\text { Alteração dos níveis de glicose sanguínea } \\
\text { Interferência na farmacodinâmica e no } \\
\text { monitoramento do nível da droga no } \\
\text { organismo }\end{array}$ \\
\hline kava-kava(Piper methysticum)Piperaceae & benzodiazepínicos & Maior efeito sedativo, coma \\
\hline valeriana(Valeriana officinalis)Valeriaceae & barbitúricos & Sedação excessiva \\
\hline $\begin{array}{l}\text { artemísia, alho, ginseng, gingko biloba, } \\
\text { gengibre }\end{array}$ & Warfarina & Alteração do tempo de coagulação \\
\hline
\end{tabular}

diterpenos podem variar, mas estas duas classes de produtos naturais, e mais nenhuma outra, devem estar sempre presentes nos óleos de copaíba ${ }^{57}$.

A prática de adulterações dos óleos de copaíba já era relatada pelos cronistas alemães em publicações do século XIX, que ensinavam como conseguir óleos sem adulterações na Amazônia Brasileira $^{58}$.

As adulterações são feitas com produtos de menor valor agregado, como óleos vegetais ou minerais (como o óleo diesel) e são comuns em toda a Região Amazônica. Os óleos adulterados podem ser encontrados nas feiras de Rio Branco (AC), no Mercado de Manaus (AM) e até em farmácias de todas as regiões do país ${ }^{56}$.

Essas adulterações foram detectadas através de estudos cromatográficos, utilizando-se cromatografia gasosa de alta resolução em fases estacionárias apolares ${ }^{6}$. Nesses estudos, observou-se que o perfil cromatográfico dos óleos adulterados é bastante diverso do perfil cromatográfico dos óleos autênticos.

Óleos de copaíba contendo diferenças no teor dos seus constituintes apresentaram atividade anti-inflamatória diferenciada, avaliada em modelos de diminuição do edema em pata de rato provocado por injeção de carragenina ou bradicinina. Os óleos de copaíba foram administrados por via oral, através de gavagem e apresentaram inibição significativa dos edemas provocados tanto por carragenina quanto por bradicinina. Desta forma, pode-se confirmar a indicação empírica dos óleos de copaíba, amplamente proclamada por silvícolas, no combate a inflamações ${ }^{7}$.

Estes estudos, entretanto, mostraram também que óleos de copaíba adulterados, ao invés de atuarem como agente anti-inflamatório, potencializaram a inflamação, provocando um aumento do volume dos edemas? ${ }^{7}$. 
Somado ao controle de qualidade que detecta adulterações, outro aspecto importante no estudo de plantas medicinais que são comercializadas como fitoterápicos é a identificação de princípios ativos responsáveis pela ação biológica da planta ${ }^{59}$.

Estudos realizados com um dos óleos de copaíba mais comuns na Região Amazônica, o óleo obtido de Copaifera multijuga, mostraram variações na composição de óleos coletados de uma mesma árvore, em períodos diversos do ano (verão-inverno) ${ }^{60}$. As substâncias detectadas foram basicamente as mesmas, mas suas concentrações variaram ${ }^{60}$. Estudos de atividade anti-inflamatória foram realizados e evidenciaram modificações nos perfis das atividades dos dois óleos ${ }^{61}$. Essas variações podem dificultar a generalização da dosagem de uso dos fitoterápicos formulados à base de óleos de copaíba.

Nos óleos de copaíba, poucos constituintes ativos identificados foram testados isoladamente, como o $\beta$-bisabolol (anti-inflamatório) ${ }^{62}$ e o $\beta$-cariofileno (bactericida ${ }^{63}$, antitumoral ${ }^{64}$ e anti-inflamatório ${ }^{65}$ ) (Figura 6).

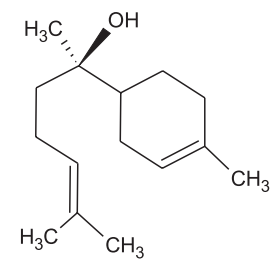

$\beta$-bisabolol

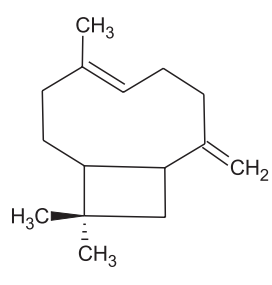

$\beta$-cariofileno
Figura 6. Estruturas de sesquiterpenos presentes em óleos de copaíba

Estudos recentes com o ácido caurenóico (Figura 7), obtido do óleo de Copaifera langsdorfii, demonstraram para este diterpeno diferentes atividades, como vasorelaxante ${ }^{66}$; relaxante do músculo liso, em estudos sobre contrações uterinas induzidas ${ }^{67}$; anti-inflamatória ${ }^{68}$; protetor de colite induzida por ácido acético ${ }^{69}$; citotóxico e embriotóxico ${ }^{70}$.

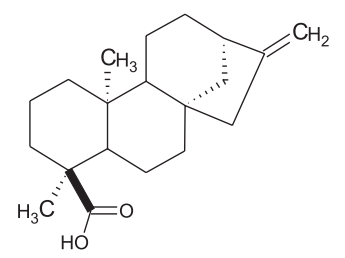

Figura 7. Estrutura do ácido caurenóico

\section{Croton cajucara Benth (Euphorbiaceae)}

Croton cajucara é uma planta nativa da região Amazônica que possui um histórico rico em benefícios terapêuticos ${ }^{14,71}$. É vulgarmente conhecido como 'sacaca' e pode ser encontrado em farmácias de produtos naturais do norte e sudeste do país, em diferentes formulações, sendo amplamente comercializado em feiras livres da região Amazônica. Tradicionalmente as cascas do caule são mais consumidas que as folhas. Estas são usadas no tratamento da diabetes, no combate a problemas gástricos, no controle da taxa de colesterol e indicadas para distúrbios do fígado (provocados pela ingestão em excesso de bebidas alcoólicas ou alimentos muito gordurosos), para ajudar na digestão. O pó das cascas é vendido em cápsulas de aproximadamente $250 \mathrm{mg}$ e o pó das folhas pode ser encontrado puro ou em mistura com o pó do boldo do Chile, sendo ambos usados no tratamento de distúrbios do fígado. É comum encontrar em lojas de produtos naturais da região norte do país (Manaus e Belém) cápsulas de cascas e folhas da sacaca em mistura com outras plantas medicinais ${ }^{14}$.

Recentemente, os benefícios terapêuticos da sacaca difundiramse por todo o país. Sua comercialização, entretanto, não preservou o uso tradicional da espécie. Foram relatados casos em que as folhas da sacaca foram vendidas com as indicações terapêuticas das cascas. Um dos autores (M. A. M. Maciel) em 1998, adquiriu em uma farmácia de manipulação localizada na Tijuca, bairro do Rio de Janeiro, um frasco contendo 25 cápsulas de folhas de sacaca, contendo no seu rótulo todas as indicações terapêuticas das cascas ${ }^{72}$.

$\mathrm{O}$ uso indevido dessa planta (folhas com indicação de cascas em tratamentos prolongados) pode causar hepatite tóxica. Pelo menos em duas capitais (Belém e Rio de Janeiro) essa planta já mostrou que pode apresentar efeitos hepáticos irreversíveis ${ }^{72}$. No Hospital Universitário da Universidade Federal do Rio de Janeiro houve um relato de falecimento (em 2001) e em Belém, na década de 1990, muitos casos de hepatite tóxica foram registrados, ocorrendo casos de óbitos. O Hospital Universitário da Universidade Federal do Pará possui um histórico significativo de pessoas vitimadas por doenças do fígado, as quais estavam ingerindo folhas em dietas prolongadas de emagrecimento. Apesar dos alertas sobre a toxicologia das folhas dessa planta veiculados pela mídia, em Belém, a população, mesmo avisada, costuma consumir as folhas para emagrecimento. Nesse caso, os usuários se dizem encorajados pelo percentual de pessoas que garantem ter emagrecido com saúde ${ }^{72}$.

Estudos realizados por nosso grupo de pesquisa destacam que o aroma e o amargor (provavelmente fornecido por diterpenos do tipo clerodano ${ }^{73,74}$ ) são bons indicadores da autenticidade de chás à base das cascas de sacaca. O clerodano trans-desidrocrotonina (DCTN, Figura 8) é o constituinte principal do chá das cascas da Sacaca, enquanto nas pílulas (pó das cascas) apresenta menor rendimento. O tratamento com o chá torna-se, portanto, um pouco mais lento (sendo necessárias duas a três semanas), que com pílulas (três vezes ao dia) uma a duas semanas. Nossos estudos usando cromatografia gasosa de alta resolução mostraram que a concentração de DCTN nas cápsulas é de 78,7\%, ligeiramente maior que no chá, com concentração de $59,9 \%^{14}$. Essa diferença se deve à baixa solubilidade da DCTN em água.

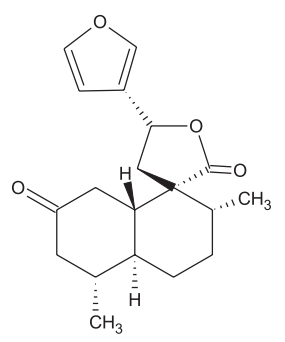

Figura 8. Estrutura da desidrocrotonina

Em um extenso projeto multidisciplinar envolvendo trabalhos etnobotânicos, fitoquímicos, químicos e farmacológicos com o Croton cajucara estão sendo verificadas as informações terapêuticas desta espécie ${ }^{75-83}$. Nesses estudos, a ação medicinal das cascas do caule da sacaca é validada pelos resultados toxicológicos obtidos, não sendo observados efeitos colaterais nos animais tratados em laboratório ${ }^{80-83}$. 


\section{ASPECTOS RECENTES NAS LEGISLAÇÕES EUROPÉIA E BRASILEIRA}

Muitos países da Europa utilizam plantas medicinais de forma ampla, como a Alemanha e a França, que detém 39 e $29 \%$ do total de vendas de toda a União Européia, respectivamente ${ }^{84}$. A legislação européia tem sido ampliada por normas, como a que determina que o marketing de produtos relacionados a ervas medicinais só possa ser veiculado mediante autorização a ser fornecida, baseada nos resultados de testes que comprovem eficácia, qualidade e segurança ${ }^{84}$. Com as regras mais restritivas, crescem as reclamações das empresas que comercializam ervas e plantas medicinais, para as quais os custos de estudos em animais e testes clínicos em humanos são quase proibitivos. Regras de ajustamento também vêm sendo emitidas para os novos países que entram na União Européia.

O Comitê da União Européia para Produtos à Base de Ervas Medicinais tem uma agenda de regulamentações mais restritiva, abrangendo a inclusão, em julho de 2007 , do controle de todas as substâncias adicionadas a alimentos ou comercializadas como suplementos alimentares, incluindo ervas, aminoácidos e ácidos $\operatorname{graxos}^{84}$

No Brasil, resoluções recentes da Agência Nacional de Vigilância Sanitária (ANVISA), de 16 de março de 2004 visam a normatização do registro de medicamentos fitoterápicos. A Resolução-RDC $\mathrm{n}^{\text {o. }} 48$ determina que todos os testes referentes ao controle de qualidade de fitoterápicos deverão ser realizados em rede credenciada no sistema REBLAS (Rede Brasileira de Laboratórios em Saúde) ou por empresas que possuam certificado de BPFC (Boas Práticas de Fabricação e Controle) $)^{85}$. Os fitoterápicos registrados antes de 31 de janeiro de 1995, com exceção daqueles já enquadrados como fitoterápicos tradicionais, deverão apresentar, no primeiro protocolo de renovação, uma série de relatórios que atestem a segurança, eficácia e as normas de produção e controle de qualidade ${ }^{85}$.

Entre as exigências da Resolução-RDC ${ }^{\circ}$. 48 estão a necessidade de controle de qualidade do produto acabado, com métodos analíticos que incluam perfis cromatográficos e resultados de prospecção fitoquímica, além de comprovação de segurança de uso, incluindo estudos de toxicidade pré-clínica. Outras resoluções da mesma data fornecem as referências bibliográficas para avaliação de segurança e eficácia de fitoterápicos $\left(\mathrm{N}^{\circ} .88\right)$, tratam dos detalhes para realização dos estudos de toxicidade $\left(\mathrm{N}^{\circ} .90\right)$ e fornecem os requisitos necessários para o processo de registro ( $\mathrm{N}^{\mathrm{o}}$.91), além da Lista de Registro Simplificado (N $\left.{ }^{\circ} 89\right)^{85}$.

\section{CONCLUSÕES}

Muitas vezes a contaminação de espécies vegetais é de caráter acidental. No entanto, a adulteração é, por definição, fraudulenta. Atualmente, não existem meios de fiscalização que garantam um controle de qualidade das ervas comercializadas. As primeiras regulamentações vêm sendo implantadas pelos órgãos de Controle Sanitário para o registro dos fitomedicamentos e fitoterápicos, mas grande parte do uso popular é baseada na comercialização em mercados e feiras populares.

Grande parte dos consumidores de plantas medicinais sentemse encorajados por acreditarem que estes remédios, por serem naturais, são inerentemente seguros. A influência da imprensa na difusão de informações errôneas sobre os efeitos das plantas medicinais é muito grande e, além disso, sem qualquer controle na maioria dos países. No Brasil é comum ouvir em propagandas a expressão: "não faz mal para a saúde porque é $100 \%$ natural". No Reino Unido e na Alemanha, onde estudos sobre a mídia têm sido reali- zados $^{86}$, comprova-se o aumento do uso de ervas medicinais pelo forte apelo de que não há contra-indicações por se tratar de produtos naturais.

Agravando ainda mais a situação, aproximadamente metade dos consumidores que utilizam plantas medicinais não avisam ao seu médico ${ }^{2}$. O nível de desconhecimento do médico só aumenta os riscos do paciente, uma vez que o médico pode errar seu diagnóstico em função das muitas interações possíveis entre as plantas e os medicamentos da medicina convencional. $\mathrm{O}$ agravamento se propaga pela disseminação dos fitofármacos da MTC, desconhecidos dos ocidentais e que, assim como os medicamentos Aiurvédicos, utilizam metais e misturas de vários extratos em suas formulações.

Cabe aos pesquisadores e à mídia, científica ou não, divulgarem os riscos a que estão expostos os consumidores que se automedicam com plantas medicinais ou fitoterápicos, sem o conhecimento necessário à sua utilização. Generalizando-se o uso seguro dos medicamentos vegetais, deve-se evitar longas terapias, já que o uso de medicação natural não significa ausência de efeitos colaterais ou tóxicos; evitar o uso associado de plantas medicinais com medicação alopata; atenção deve ser dada aos produtos naturais de origem chinesa e indú, já que há possibilidade da presença de metais; deve-se adquirir o vegetal de fontes seguras; indivíduos mais vulneráveis (crianças, mulheres grávidas ou em lactação) devem evitar o consumo de plantas medicinais e, finalmente, seguindo estes passos, se houver efeitos adversos, deve-se interromper o uso do medicamento e buscar ajuda médica.

Estudos multidisciplinares envolvendo etnobotânicos, químicos, farmacólogos e agrônomos (neste caso, no controle do cultivo de ervas medicinais) são necessários para que sejam ampliados os conhecimentos das plantas medicinais, como agem, quais são os seus efeitos tóxicos e colaterais, como seriam suas interações com novos medicamentos alopatas e quais as estratégias mais adequadas para o controle de qualidade e produção de fitoterápicos, atendendo às novas normas das agências reguladoras, como as resoluções da ANVISA.

\section{AGRADECIMENTOS}

Os autores agradecem à CAPES e à FAPERJ.

\section{REFERÊNCIAS}

1. Akerele, O.; Herbal Gram 1993, 28, 13.

2. Eisenberg, D.; J. Am. Med. Assoc. 1998, 280, 1569.

3. Calixto, J. B.; Braz. J. Med. Biol. Res. 2000, 33, 179.

4. Blumentahl, M., ed.; The Complete German Commission E Monographs: Therapeutic Guide to Herbal Medicines; American Botanical Council: New York, 1998.

5. Bulletin of the World Health Organization. Regulatory situation of herbal medicines. A worldwide review, Geneva, 1998.

6. Veiga Jr., V. F.; Pinto, A. C.; Patitucci, M. L.; Quim. Nova 1997, $20,612$.

7. Veiga Jr., V. F.; Pinto, A. C.; Patitucci, M. L.; Zanino, L.; Calixto, J. B.; Phytother. Res. 2001, 15, 476.

8. Perharic, L.; Shaw, D.; Murray, V.; Lancet 1993, 342, 180.

9. Simões, M. O.; Schenkel, E. P.; Gosmann, G.; Mello, J. C. P.; Mentz, L A.; Petrovick, P. R., orgs.; Farmacognosia: da planta ao medicamento; Ed. Universidade/UFRGS/ Ed. da UFSC: Porto Alegre/ Florianópolis, 1999.

10. Schaller, M.; Korting, H. C.; Clin. Exp. Dermatol. 1995, 20, 143.

11. Capasso, R.; Izzo, A. A.; Pinto, L.; Bifulco, T.; Vitobello, C.; Mascolo, N.; Fitoterapia 2000, 71, S58.

12. Guil, J.L.; Rodriguez-Garcia, I.; Torija, E.; Plant Foods Hum. Nutr. 1997, $51,99$.

13. Ames, B.N.; Science 1983, 221, 1256.

14. Maciel, M. A. M.; Pinto, A. C.; Veiga Jr., V. F.; Martins, J. R.; Grynberg, N. F.; Echevarria, A.; Lapa, A. J.; Vanderlinde, F. A.; Phytochem. Pharmacol. II Ser. Recent Prog. Med. Plants 2002, 8, 460.

15. Loeper, J.; Descatoire, V.; Letteron, P.; Moulis, C.; Degott, C.; Dansette, P.; Fau, D.; Pessayre, D.; Gastroenterol. 1994, 106, 464. 
16. Fau, D. ; Lekehal, M. ; Farrell, G. ; Moreau, A. ; Feldmann, G. ; Haouzi, D. ; Pessayre, D. ; Gastroenterol. 1997, 113, 1334.

17. Tyler, V. E.; Chemtec 1997, 5, 52.

18. Buckel, P.; Naturwissenschaften 1998, 85, 155.

19. Simões, C. M. O.; Mentz, L. E.; Schenkel, E. P.; Irgang, B. E.; Stehmann, J. R.; Plantas da Medicina Popular do Rio Grande do Sul, Eduni-Sul: Porto Alegre, 1986.

20. Hoene, F. C.; Plantas e substância vegetais tóxicas e medicinais, Graphicars: São Paulo, 1939.

21. Secco, R. S.; Ciência e Cultura 1990, 42, 807.

22. Davis, P.; Aromaterapia, Ed. Martins Fontes: São Paulo, 1996.

23. Oliveira M. G. M.; Monteiro, M. G.; Macaúbas, C.; Barbosa, V. P.; Carlini, E. A.; J. Ethnopharmacol. 1991, 34, 29.

24. Souza-Formigoni, M. L. O.; Oliveira, M. G. M; Monteiro, M. G.; SilveiraFilho, N. G.; Braz, S.; Carlini, E. A.; J. Ethnopharmacol. 1991, 34, 21.

25. Jorge, R. M.; Leite, J. P. V.; Oliveira, A. B.; Tagliati, C. A.; J. Ethnopharmacol. 2004, 94, 93.

26. Montanari, T. ; Bevilacqua, E.; Contraception 2002, 65, 171.

27. Ernst, E.; Trends Pharmacol. Sci. 2002, 23, 136.

28. Shaw, D.; Drug Saf. 1997, 17, 342.

29. Itankar, P. R. ; Hamdard Med. 2001, 19, 95.

30. Ernst, E.; J. Clin. Pharmacol. Ther. 2001, 70, 497.

31. Koh, H. L.; Woo, S. O.; Drug Saf. 2000, 23, 351.

32. Ko, R. J.; New Engl. J. Med. 1998, 339, 847.

33. Caldas, E. D.; Machado, L. L.; Food Chem. Toxicol. 2004, 42, 599

34. Ernst, E: Am. J. Med. 1998, 104, 170

35. Josefson, D.; Brit. Med. J. 1996, 312, 1378.

36. Doyle, H.; Kargin, M.; Brit. Med. J. 1996, 313, 756.

37. Graham-Brown, R. A. C.; Bourke, J. F.; Bumphrey, G.; Brit. Med. J. 1994, $308,473$.

38. Cosyns, J.P.; Am. J. Kidney. Dis. 1999, 33, 1011.

39. Woolf, G. M.; Petrovick, L. M.; Rojter, S. E.; Ann. Int. Med. 1994, 121, 729.

40. Nortier, J. L.; New Engl. J. Med. 2000, 342, 1686.

41. Kao, W. F.; Hung, D. Z.; Lin, K. P.; Hum. Exp. Toxicol. 1992, 11, 480.

42. But, P. P. H.; Tomlinson, H.; Cheung, K. O.; Yong, S. P.; Szeto, M. L.; Lee, C. K., Brit. Med. J. 1996, 313, 117.

43. Bowen, I. H.; Cubbin, I. J. Em Adverse effects of herbal drugs; De Smet, P. A. G. M.; Keller, K.; Hansel, R.; Chandler, R. F., eds.; Springer Verlag: Berlin, 1993.

44. Chang, L.W.; Yang, C. M.; Chen, C. F.; Deng, J. F.; Biomed. Environ Sci. 1992, 5, 283.

45. Ahmed, M.; Bull. Envirom. Contam. Toxicol. 2001, 66, 421.

46. Jones, N.; New Sci. 2001, 3, 10.

47. Czech, E.; Kneifel, W.; Kopp, B.; Planta Med. 2001, 67, 263.

48. Robbers, J. E.; Speedie, M. K.; Tyler, V. E.; Farmacognosia e Farmacobiotecnologia, Tradução Benedetti, I. C.; Editorial Premier: São Paulo, 1997, cap. 9.

49. Mehta, D.K.; British National Formulary. British Medical Association and the Royal Pharmaceutical Society of Great Britain, The Pharmaceutical Press: London, 1998.

50. Newall, C. A.; Anderson, L. A.; Phillipson, J. D.; Herbal Medicines: A guide for health-care professionals, The Pharmaceutical Press: London, 1996.

51. Piscitelli, S. C.; Burstein, A. H.; Welden, N.; Gallicano, K. D.; Falloon, J.; Clin. Infec. Dis. 2002, 34, 234.

52. Di Carlo, G.; Borreli, F.; Ernst, E.; Izzo, A. A.; Trends Pharmacol. Sci. 2001, 22, 292

53. Henney, J. E.; J. Am. Med. Assoc. 2000, 13, 283.

54. Williamson, E. M.; Phytomed. 2001, 8, 401.

55. Miller, I. G.; Arch. Intern. Med. 1998, 158, 2200
56. Veiga Jr., V. F.; Pinto, A. C.; Quim. Nova 2002, 25, 273.

57. Veiga Jr., V. F.; Tese de Doutorado, Universidade Federal do Rio de Janeiro, Brasil, 2004.

58. Freise, F. W.; Süddeut. Apot-Ztg. 1937, 77, 11.

59. Maciel, M. A. M.; Echevarria, A.; Veiga Jr, V. F.; Pinto, A. C.; Quim. Nova 2002, 25, 429.

60. Veiga Jr., V. F.; Tese de Mestrado, Universidade Federal do Rio de Janeiro, Brasil, 1998.

61. Veiga Jr., V. F.; Zunino, L.; Calixto, J. B.; Patitucci, M. L.; Pinto, A. C.; Planta Med., no prelo.

62. Zekovic, Z.; Pekie, B.; Lepojevic, Z.; Petrovic, L.; Chromatographia 1994, 39,587

63. Kang, R.; Helms, R.; Stout, M. J.; Jaber, H.; Nakatsu, T.; J. Agric. Food Chem. 1992, 40, 2328.

64. Zheng, G.Q.; Kenney, P. M.; Lam, L. K. T.; J. Nat. Prod. 1992, 55, 999.

65. Shimizu, M.; Shogawa, H.; Matsuzawa, T.; Yonezawas, S.; Hayashi, T.; Arisawa, M.; Suzuki, S.; Yoshizaki, M.; Morita, N.; Chem. Pharm. Bull. 1990, 38, 2283.

66. Tirapelli, C. R.; Ambrosio, S. R.; Costa, F. B.; Coutinho, S. T.; Oliveira, D. C. R.; Oliveira, A. M.; Eur. J. Pharmacol. 2004, 492, 233.

67. Cunha, K. M. A.; Paiva, L. A. F.; Santos, F. A.; Gramosa, N. V.; Silveira, E. R.; Rao, V. S.; Phytother. Res. 2003, 17, 320

68. Paiva, L. A. F.; Gurgel, L. A.; Silva, R. M. A.; Tomé, R.; Gramosa, N. V.; Silveira, E. R.; Santos, F. A.; Rao, V. S. N.; Vasc. Pharm. 2002, 39, 303.

69. Paiva, L. A. F.; Gurgel, L. A.; Souza, E. T.; Silva, R. M. A.; Tomé, R.; Silveira, E. R.; Santos, F. A.; Rao, V. S. N.; J. Ethnopharmacol. 2004, 93, 51.

70. Costa-Lotufo, L. V.; Cunha, G. M. A.; Farias, P. A. M.; Viana, G. S. B.; Cunha, K. M. A.; Pessoa, C.; Moraes, M. O.; Silveira, E. R.; Gramosa, N. V.; Rao, V. S.; Toxicon 2002, 40, 1231.

71. Maciel, M. A. M.; Pinto, A. C.; Arruda, A. C.; Pamplona, S. G. S. R.; Vanderlinde, F. A.; Lapa, A. J.; Cólus, I. M. S.; Echevarria, A.; Grynberg, N. F.; Farias, R. A. F.; Luna Costa, A. M.; Rao, V. S. N.; J. Ethnopharmacol. 2000, 70, 41 .

72. Maciel, M. A. M.; trabalho não publicado.

73. Maciel, M. A. M.; Pinto, A. C.; Brabo, S. N.; Silva, M. N.; Phytochemistry 1998, 49, 823

74. Maciel, M. A. M.; Pinto, A. C.; Kaiser, C. R.; Magn. Reson. Chem. 2003, $41,278$.

75. Van Den Berg, M. E.; Plantas Medicinais na Amazônia- Contribuição ao seu Conhecimento Sistemático, Gráfica Editora Falangola: Belém, 1982.

76. Silva, R. M.; Santos, F. A.; Maciel, M. A. M.; Pinto, A. C.; Rao, V. S.; Planta Med. 2001, 67, 763.

77. Silva, R. M.; Santos, F. A.; Maciel, M. A. M.; Pinto, A. C.; Rao, S. N.; Pharm. Pharmacol. 2001, 53, 535.

78. Luna Costa, A. M.; Silva, J. C. R.; Campos, A. R.; Rao, V. S. N.; Maciel, M. A. M.; Pinto, A. C.; Phytother. Res. 1999, 13, 689.

79. Grynberg, N. F.; Echevarria, A.; Lima, J. E.; Pamplona, S. G. S. R.; Pinto, A. C.; Maciel, M. A. M.; Planta Med. 1999, 65, 687.

80. Âgner, A. R.; Maciel, M. A. M.; Pinto, A. C.; Pamplona, G. R. S.; Cólus, I. M. S.; Terat., Carcinog., Mutag. 1999, 19, 377.

81. Âgner, A. R.; Maciel, M. A. M.; Pinto, A. C.; Cólus, I. M.; Planta Med. 2001, 67, 815 .

82. Campos, A. R.; Albuquerque, F. A. A.; Rao, V. S. N.; Maciel, M. A. M.; Pinto, A. C.; Fitoterapia 2002, 73, 116.

83. Rodríguez, J. A.; Haun, M.; Planta Med. 1999, 65, 522.

84. Silano, M.; De Vincenzi, M.; De Vincenzi, A.; Silano, V.; Fitoterapia 2004, $75,107$.

85. http://e-legis.bvs.br/leisref/public/showAct.php?id=10230, acessada em Outubro 2004.

86. Ernst, E.; Weihmayr, T.; Br. Med. J. 2000, 321, 707 